\title{
Frustration Measure for Molecular Magnets with Single-Ion Anisotropy
}

\author{
P. KozŁOWSKI* \\ Faculty of Physics, Adam Mickiewicz University in Poznań, Umultowska 85, 61-614 Poznań, Poland \\ The universal frustration measure and its extension to anisotropic systems is calculated for a family of spin \\ Hamiltonians modeling a hypothetical ring-shaped chromium-based molecular magnets. The proposed extension \\ takes into account the impact of single-ion anisotropy on the magnetic centers and behaves more consistently with \\ respect to the isotropic counterpart. The influence of anisotropy on both measures is assessed, which leads to a \\ conclusion that frustration decreases with increasing absolute value of single ion anisotropy.
}

DOI: $10.12693 /$ APhysPolA.133.423

PACS/topics: 75.45.+j, 75.50.Xx, 75.75.-c, 03.67.Mn

\section{Introduction}

Molecular magnets are molecules containing magnetic ions. They have been studied for years because of their potential technological applications e.g. in quantum computing [1], or information storage [2]. However, a lot of research on this kind of molecules is also motivated by purely scientific interests. Since magnetic molecules, which are obtained by chemical methods, are shielded from each other by non-magnetic ligands intermolecular magnetic interactions are practically negligible. Therefore measurements made on bulk samples reflect properties of a single molecule. Moreover, phenomena characteristic for bulk magnetic materials can also be observed in molecular magnets, though they have usually different characteristics due to the nanoscopic size of investigated objects. A small size of these molecules make them accessible for theoretical modeling and thus they can be a perfect test bed for investigation of various phenomena that are hard to model in bulk materials.

One of such phenomena is magnetic frustration that has been first analyzed in the context of classical spin glasses [3], but appears also in quantum systems [4]. Recently frustration effects have been studied in nanomagnets [5]. Of various frustrated molecules the oddmembered antiferromagnetic rings seem to be the most suitable for analysis of frustration due to their simplicity and the existence of various experimental examples [6-9]. Among them the family of 9-membered chromium rings is probably the most studied in this context since these molecules were used to classify frustration types [9], assess frustration signatures [10] and to verify a link between frustration and entanglement [11].

Nonetheless there is no study focused on the dependence of frustration on anisotropy. Such dependence can be conjectured because anisotropy can be considered as a kind of spacial restraint similar to that which causes

*e-mail: kozl@amu.edu.pl so called geometric frustration (e.g. triangle shapes in antiferromagnetically coupled network of magnetic ions). Besides, anisotropy plays a crucial role in molecular magnetism and is present in most of nanomagnets. In this paper the relation between frustration and single-ion anisotropy is investigated in a quantum spin ring that models antiferromagnetically coupled magnetic ions in a ring shaped molecule. Some extension of the existing frustration measures is proposed to take into account the influence of single-ion anisotropy.

\section{Spin model and frustration measures}

Homometalic molecular quantum spin rings can be modeled by the following spin Hamiltonian:

$$
\mathcal{H}=\sum_{j=1}^{n-1} J s_{j} \cdot s_{j+1}+\alpha J s_{1} \cdot s_{n}+\sum_{j=1}^{n} D\left(s_{j}^{z}\right)^{2},
$$

with the exchange and anisotropic terms respectively. For for the family of chromium rings $s$ stands for $s=3 / 2$ quantum spin operator, $J=16.6 \mathrm{~K}, D=-0.34 \mathrm{~K}, n=9$ and the value of $\alpha$ depends on the particular ring considered $[9,12,13]$. However to save time needed for calculations in what follows it will be assumed that $n=7$ which corresponds to hypothetical chromium rings. The results obtained in [11] suggest that there is no qualitative difference between $n=7$ and $n=9$ cases. Thus, when changing $\alpha$ from negative to positive values one can smoothly switch on geometric frustration $(\alpha>0)$ and model all the hypothetical 7-membered chromium rings.

Frustration in the ground state can be measured for each pair $\mathrm{S}$ of the interacting spins with the help of the following universal frustration measure [14]:

$$
f_{S}=1-\operatorname{Tr}\left(\rho_{G} \Pi_{S} \otimes 1_{R}\right)=1-\operatorname{Tr}\left(\rho_{S} \Pi_{S}\right)
$$

where $\rho_{G}$ is a ground state of a total system $(T=S \cup R)$ described by Hamiltonian (1), $\Pi_{S}$ is the projector into the ground space of the smallest interacting subsystem $S, 1_{R}$ is the unity operator with the dimension equal to that of the Hilbert space of the rest of the system $(R)$, and 
$\rho_{S}=\operatorname{Tr}_{R}\left(\rho_{G}\right)$.

Thus, the interaction $S$ is frustrated if $f_{S}$ is larger than 0 , with the maximal frustration reached for $f_{S}=1$. For the non-frustrated pair interaction $f_{S}=0$. If $D=0$ the smallest interacting system is a pair of nearest neighbors coupled by exchange interaction. Then $f_{S}$ for any other pair is equal to 0 . However for anisotropic systems those spins which are not connected through exchange interactions are always exposed to the same single-ion anisotropy. As a result a ground state space of a separated pair of such spins is more restricted (smaller degeneration of the ground state) than in the isotropic case leading to $f_{S} \neq 0$. Thus, when calculating a mean value of $f_{S}$, measuring a mean frustration of an entire system, it seems reasonable that for a molecule with single-ion anisotropy also pairs of spins not coupled by exchange interaction are taken into account. In what follows two mean measures are considered:

$$
f=\frac{1}{n} \sum_{S=1}^{n} f_{S}, \quad f_{\text {tot }}=\frac{1}{n(n-1) / 2} \sum_{S=1}^{n(n-1) / 2} f_{S},
$$

The former is an average over exchange interacting pairs (here nearest neighbors), which is the original definition of $f$ [14] and the latter an average over all pairs of spins. For Hamiltonian (1) systems $\mathrm{S}$ are described by the local Hamiltonians

$$
h_{i}=J_{i j} s_{i} \cdot s_{j}+D\left[\left(s_{i}^{z}\right)^{2}+\left(s_{j}^{z}\right)^{2}\right],
$$

if the spins are coupled by exchange interaction, with $J_{i j}=J$ for all the pairs of nearest neighbors $(j=i+1$ with cyclic boundary conditions) but $(n, 1)$ for which $J_{n 1}=\alpha J$ and by

$$
h_{i j}=D\left[\left(s_{i}^{z}\right)^{2}+\left(s_{j}^{z}\right)^{2}\right],
$$

for the remaining pairs of spins.

It should be noticed that the ground state of Hamiltonian (1) is degenerated. In this case measure $f_{S}$ is calculated for the so called maximally mixed ground state, which is a mixed state composed of all degenerated ground states [15]. All ground states have been calculated numerically with the help of a simple power method. The fast convergence was ensured by the use of a good starting point generated like in Ref. [11] with the help of the Suzuki-Trotter formula and exploiting the elements of the quantum transfer matrix approach.

\section{Results}

In Fig. 1 frustration $f_{\text {tot }}$ is shown only for anisotropic system because for isotropic one these quantities differ only by a multiplicative factor $f_{\text {tot }}=2 f /(n-1)$. One can distinguish three regions where frustration takes different values: I for $-1<\alpha<0$, II for $0<\alpha<\alpha_{c}$ and III for $\alpha_{c}<\alpha<2$, with $\alpha_{c}$ depending on the value of anisotropy $D$. The sudden jumps at $\alpha=0$ are due to the change of the ground state of the pair of spins bounded by coupling $\alpha J$ and at $\alpha=\alpha_{c}$ are due to a change of a global ground state [11]. What is important here are different values of $f$ and $f_{\text {tot }}$ for an anisotropic

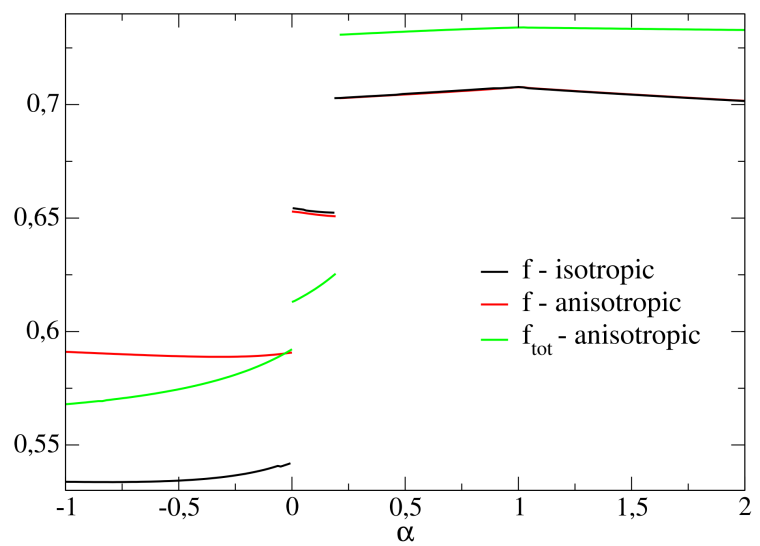

Fig. 1. Mean frustration for an isotropic and anisotropic $(D=-0.34 \mathrm{~K}) \quad 7$-membered spin ring as a function of $\alpha$.

system. In region I $f$ is almost constant, whereas $f_{t o t}$ though smaller than $f$ is growing and just before $\alpha=0$ intersects with $f$ meaning that at that point an average frustration of exchange coupled spin pairs is the same as average frustration of the remaining spin pairs. In region II and III $f_{\text {tot }}$ is below respectively above $f$ which means that not exchange bonded spin pairs are less respectively more frustrated than the bonded ones. Compared to the isotropic case $f$ for an anisotropic ring is definitely larger in region I and very similar in regions II and III. $f_{\text {tot }}$ for an anisotropic ring is always larger than $f_{\text {tot }}$ for isotropic one since isotropic $f_{\text {tot }}$ is here equal to isotropic $f / 3$.

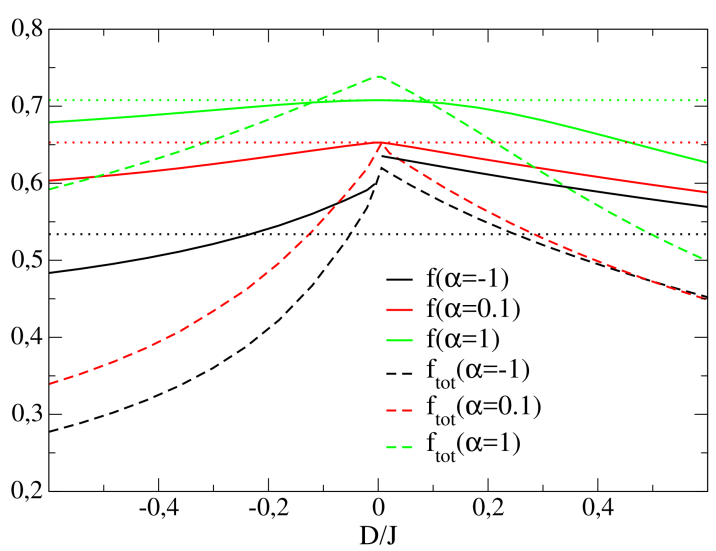

Fig. 2. Mean frustration for some values of $\alpha$ for a 7membered spin ring as a function of $D$. The values of $f$ for $D=0$ are shown with dotted curves in corresponding colors.

To estimate the influence of anisotropy strength on frustration, values of $f$ and $f_{\text {tot }}$ at points $\alpha=-1,0.1$ and 1 corresponding to three plateaus in regions I, II and III respectively are shown in Fig. 2 versus anisotropy $D$. It can be seen (Fig. 2) that $f$ and $f_{\text {tot }}$ are decreasing with increasing absolute value of anisotropy $D$ though $f_{\text {tot }}$ is decreasing much faster. It means that $f_{S}$ decreases faster 
for spins that are not exchange coupled. For sufficiently small negative values of $D f(\alpha=-1)$ intersects with the isotropic counterpart (dotted and full black curves in Fig. 2) which means that for strong negative single-ion anisotropy $f$ is smaller than in the isotropic system in all three regions. $f_{\text {tot }}$ for anisotropic ring remains larger than the isotropic counterpart for all values of $D$ in the studied range. Moreover, the intersection of green broken and full curves means that the relation between $f$ and $f_{\text {tot }}$ in region III depends on the value of anisotropy $D$. Finally it can be noticed that for sufficiently large positive value of $D$ black and red broken curves intersect which means that a clear-cut picture of three distinct plateaus shown in Fig. 1 is not any more valid, though the jumps at points $\alpha=0$ and $\alpha=\alpha_{c}$ are still observed.

The decrease of $f$ and $f_{\text {tot }}$ with increasing absolute value of $D$ can be understood as the result of an increasing role played by the single-ion anisotropy. With larger $D$ the ground state of Hamiltonian (1) is more determined by the value of $D$ and depends less on the exchange interactions. Thus, the exchange interactions between the chosen spin pair $S$ and the rest of the system have smaller influence on the reduced ground state $\rho_{S}$. Therefore the overlaps between state $\rho_{S}$ and the ground state density matrices for Hamiltonians (5) or (6) are larger leading to smaller values of $f_{S}$. It can be expected that in the limit $D / J \rightarrow \infty$ the values of $f$ and $f_{\text {tot }}$ coincide.

\section{Conclusions}

The universal frustration measures $f$ and its extension $f_{\text {tot }}$ have been calculated for a 7 -membered $s=3 / 2$ spin ring modeling a hypothetical chromium nanomagnet. It has been demonstrated that these measures differ from each other. Their dependence on values of anisotropy $D$ has been investigated leading to a conclusion that the inclusion of anisotropy always increases $f_{\text {tot }}$, though this increase is smaller for larger absolute values of $D$. For measure $f$ the influence of anisotropy depends on values of $\alpha$ and $D$, though like in the case of $f_{\text {tot }}$ also $f$ decreases with increasing absolute value of $D$. It seems that $f_{\text {tot }}$ is a more appropriate frustration measure for anisotropic systems as it takes into account non-exchange coupled spin pairs and behaves more consistently with respect to the isotropic case. It would be interesting to apply measure $f_{\text {tot }}$ to systems in magnetic field.

\section{Acknowledgments}

Part of the calculations have been carried out at Poznań Supercomputing and Networking Center in Poznań, Poland.

\section{References}

[1] B. Georgeot, F. Mila, Phys. Rev. Lett. 104, 200502 (2010).

[2] M. Mannini, F. Pineider, P. Sainctavit, C. Danieli, E. Otero, C. Sciancalepore, A.M. Talarico, M.-A. Arrio, A. Cornia, D. Gatteschi, R. Sessoli, Nature Mater. 8, 194 (2009).

[3] Spin Glass Theory, Beyond, Eds.: M. Mézard, G. Parisi, M.A. Virasoro, Word Scientific, Singapore 1987.

[4] Introduction to frustrated magnetism, Eds. C. Lacroix, F. Mila, P. Mendels, Springer, Berlin, Heidelberg 2010).

[5] J. Schnack, Dalton Transactions 39, 4677 (2010).

[6] O. Cador, D. Gatteschi, R. Sessoli, A.-L. Barra, G. A. Timco, R.E.P. Winpenny, J. Magn. Magn. Mater. 290-291, 55 (2005).

[7] H. C. Yao, J. J. Wang, Y. S. Ma, O. Waldmann, W. X. Du, Y. Song, Y. Z. Li, L. M. Zheng, S. Decurtins, X. Q. Xin, Chem. Commun. 16, 1745 (2006).

[8] N. Hoshino, M. Nakano, H. Nojiri, W. Wernsdorfer, H. Oshio, J. Am. Chem. Soc. 131, 15100 (2009).

[9] M.L. Baker, G.A. Timco, S. Piligkos, J.S. Mathieson, H. Mutka, F. Tuna, P. Kozłowski, M. Antkowiak, T. Guidi, T. Gupta, H. Rath, R.J. Woolfson, G. Kamieniarz, R.G. Pritchard, H. Weihe, L. Cronin, G. Rajaraman, D. Collison, E.J.L. McInnes, R.E.P. Winpenny, Proc. Nat. Acad. Sci. 109, 19113 (2012)

[10] P. Kozłowski, M. Antkowiak, G. Kamieniarz, J. Nanopart. Res. 13, 6093 (2011).

[11] P. Kozłowski, Phys. Rev. B 91, 174432 (2015).

[12] M. Antkowiak, P. Kozłowski, G. Kamieniarz, G.A. Timco, F. Tuna, R.E.P. Winpenny, Physical Review B 87, 184430 (2013).

[13] R. J. Woolfson, G. A. Timco, A. Chiesa, I.J. Vitorica Yrezabal, F. Tuna, T. Guidi, E. Pavarini, P. Santini, S. Carretta, R.E.P. Winpenny, Ang. Chem. Int. Ed. 55, 8856 (2016).

[14] S. M. Giampaolo, G. Gualdi, A. Monras, F. Illuminati, Phys. Rev. Lett. 107, 260602 (2011).

[15] U. Marzolino, S. M. Giampaolo, F. Illuminati, Phys. Rev. A 88, 020301 (2013). 